\title{
REMOTE MONITORING AND INTELLIGENT FAILURE DIAGNOSIS OF ROTATING MACHINERY USING THE B/S MODE
}

\author{
Jianping $\mathrm{He}$ \\ Chongqing College of Finance and Economics, Yongchuan 402160, Chongqing, China. \\ Email: hejianpingcyc@yeah.net
}

\begin{abstract}
In order to reduce the failure wear of rotating machinery, the subsequent economic loss and casualties, the remote vibration monitoring and fault diagnosis technology of rotating machinery based on B/S (browser/server) mode is studied. Firstly, the characteristics and structure of $\mathrm{B} / \mathrm{S}$ mode, the remote vibration monitoring and fault diagnosis system of rotating machinery are analyzed and discussed. And then the system structure, flow chart, server and client design and implementation method are displayed. Subsequently, the overall scheme of rotating machinery fault diagnosis system based on B/S structure is designed. Furthermore, based on the idea of modularization, the scheme design of each module of the system is carried out, including network data acquisition and simulation experiment. Finally, the data and related information in the process of fault diagnosis are stored by analyzing the performance of database server, map refresh display and rotor phase. The remote monitoring system based on $\mathrm{B} / \mathrm{S}$ can objectively reflect the vibration signal of rotating machinery, obtain the industrial field data in time, analyze the operation status of the equipment, find and correct the unreasonable elements in the design, and also show new design requirements and develop new products according to the actual needs of the site. It is helpful for on-site monitoring personnel to carry out routine monitoring and diagnosis of the equipment, saving about half of the human and material resources, and the results of remote monitoring are relatively accurate, which can diagnose the fault in time. Remote monitoring and fault diagnosis provide the first-hand information from the scene for scientific research institutes, and combine scientific research and production practice more closely. As a whole, large-scale rotating machinery is the key equipment in the production unit of enterprises. It is important to provide remote monitoring and fault diagnosis for enterprises. The achievements of remote monitoring and fault diagnosis system greatly improve the production efficiency.
\end{abstract}

Keywords: B/S mode; Remote; Rotating machinery; Vibration monitoring; Fault diagnosis; Database.

\section{Introduction}

With the maturity of Web technology, the complexity of distributed system is increasing. The network technology is also integrated into monitoring and diagnosis system and plays an essential role in the safe production. For real-time monitoring and diagnosis system, there are two popular structures: $\mathrm{C} / \mathrm{S}$ (client/server) structure and B/S structure. B/S mode is more and more widely used because of its good characteristics.

The key of vibration monitoring is the analysis of vibration signal, and it is very important to choose the signal analysis method. Generally, the stationary signal is usually analyzed by Fourier analysis method, but in the actual monitoring, the systemresponse signal is usually nonlinear and nonstationary [1]. Therefore, the signal processing is mainly to study the nonlinear and non-stationary signals. Although the development of diagnosis technology makes great progress and many cases are successfully implemented by using it, it still cannot meet the actual needs of the rapid development of the market. And there is still a long way to go for the new fault diagnosis methods, technologies and systems [2]. Rotating machinery fault diagnosis technology is a very complicated and multidisciplinary technology.

The technology is a collection of many disciplines, including PC (personal computer), sensor and detection technology, signal processing, fault identification, system identification, optimization algorithm, and vibration [3]. In a sense, despite great achievements and progress, the fault diagnosis technology of rotating machinery lacks systematicness, scientificity and integrity. Hence, the further studies on new technology and new methods of fault diagnosis should be carried out.

Design and implementation of remote monitoring and diagnosis system for rotating machinery based on B/S structure are as follows: Firstly, sensors are installed on the equipment site to extract the original 
vibration signal data. Afterwards, the signal is filtered and extracted by the equipment monitoring substation, and transmitted to the remote database server and Web server. Remote users can view the analysis of real-time monitoring data and historical trend data through IE (internet explorer) browser. When the monitoring value of the equipment is wrong, the alarm will ring. According to the analysis of real-time and historical data, mechanical equipment experts can carry out remote troubleshooting [4]. The remote monitoring and fault diagnosis of rotating machinery breaks through geological restraint, and all levels of personnel in the enterprise can monitor the operation status of the equipment anytime and anywhere through the network, saving manpower and material resources, and improving the economic benefits of the enterprise [5]. And the open structure makes the rich diagnosis resources shared, promotes the further improvement of the diagnosis knowledge base and database, and improves the overall diagnosis ability of the equipment monitoring and diagnosis system [6]. Enterprises, equipment manufacturers and scientific research institutions form a fault diagnosis alliance, strengthening their technical cooperation and achieving win-win results [7]. Therefore, the development of remote equipment monitoring and diagnosis system has very important significance in theory and practice. And it will has a promising future.

\section{B/S Structure and Remote Monitoring and Diagnosis Technology \\ 2.1 B/S Structure}

The traditional C/S structure is developed with the application of network database technology. The structure of C/S model first appears in the 1980s. The general two-tier structure, namely "fat client" structure, is a typical and common form. This form of $\mathrm{C} / \mathrm{S}$ structure is divided into two layers: the first layer combines the user interface and business logic in the client system; the second layer combines the database server through the network, and the system tasks are completed by the client and the server respectively. In the $\mathrm{C} / \mathrm{S}$ two-tier structure, the client keeps the application program and directly accesses the database; the server keeps all the data; each client keeps a trust connection with the database. The client sends a request to the data server through the application program. The data server operates the database according to the request and returns the corresponding result to the client. The server has the functions of data acquisition, control and communication with the client; the client communicates with the server and user interface module [8]. C/S structure separates a complex network application from a vivid and intuitive user interface, leaving a large number of data operations to the background to process, which improves the speed of user interaction. The development and application process are simple, and the development tools are very applicable, which plays a greater role in promoting the application of network database. The $\mathrm{C} / \mathrm{S}$ structure is shown in Figure 1.

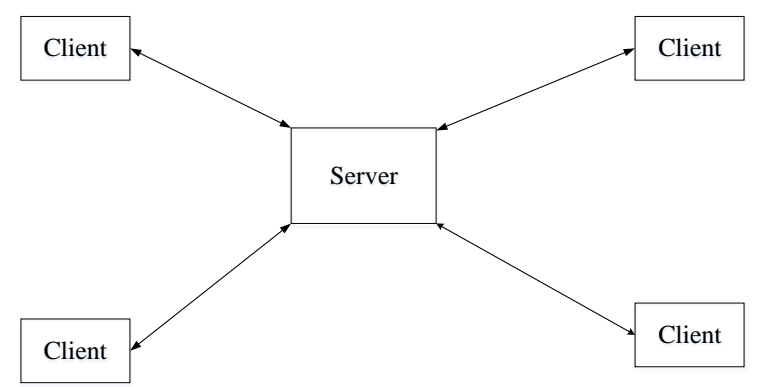

Figure 1: C/S structure

Because of these shortcomings of $\mathrm{C} / \mathrm{S}$ structure, a layer is added in the middle of the traditional $\mathrm{C} / \mathrm{S}$ structure, and the functions of the original client are given to the middle layer, which is the web server layer. In this way, the client is not responsible for the original data storage, and the browser is just needed to be installed on the client. The original server is taken as the database server, on which the database management system is installed and the database is created. The function of Web server is to access the database and transfer it to the browser through Internet/Intranet. Thus, the Web server is not only the server of the browser, but also the browser of the database server. In this mode, the client becomes a simple browser, forming a "fat server/thin client" mode, which is the $\mathrm{B} / \mathrm{S}$ mode. The structure based on $\mathrm{B} / \mathrm{S}$ mode combines Web with database to form a Web computing mode. The mode is applied to the Internet to form a three-tier client/server application structure. The structure clearly divides the three functional levels of the application system, making them logically independent [9]. Its structure is shown in Figure 2.

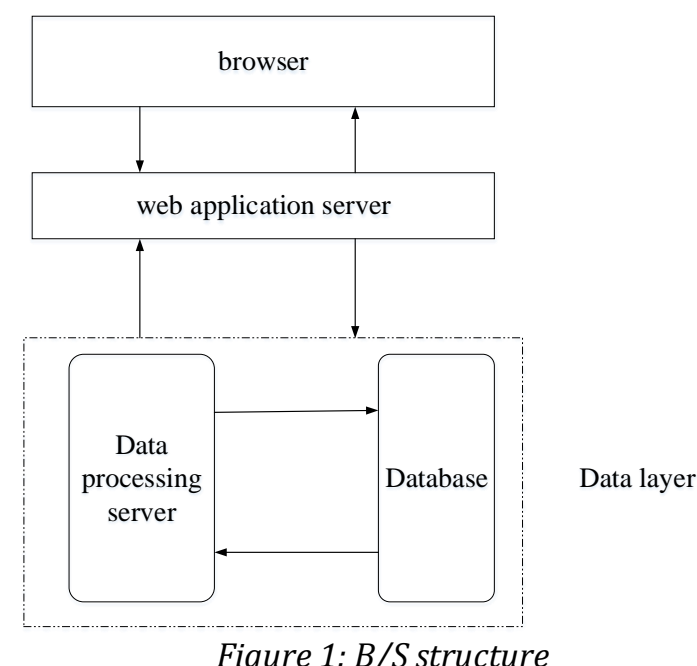

Figure 1: $B / S$ structure 
With the rapid development of the Internet, the system cannot be limited to certain regions but needs to have greater scalability for the remote monitoring and diagnosis. Hence, B/S structure come into being, and its structure is an improvement of $\mathrm{C} / \mathrm{S}$ structure. $\mathrm{B} / \mathrm{S}$ mode adopts three-tier structure, namely client, server and database server. The client only uses the browser to access the server, the transaction processing is on the server, and the data processing is carried out by the database server, so that the remote data acquisition can be carried out through the network. But only in B/S mode, it can't communicate with the controlled object directly [10]. The remote real-time monitoring system based on $\mathrm{B} / \mathrm{S}$ mode to realize data communication with the controlled object needs to adopt a four-layer structure, namely the presentation layer, the function layer, the data layer and the control layer. As shown in the figure, compared with the traditional $\mathrm{C} / \mathrm{S}$ structure, it appears that just the control service layer is added to the three-tier B/S structure, which is used to communicate with the web server and control the controlled object. In fact, it is not the case.

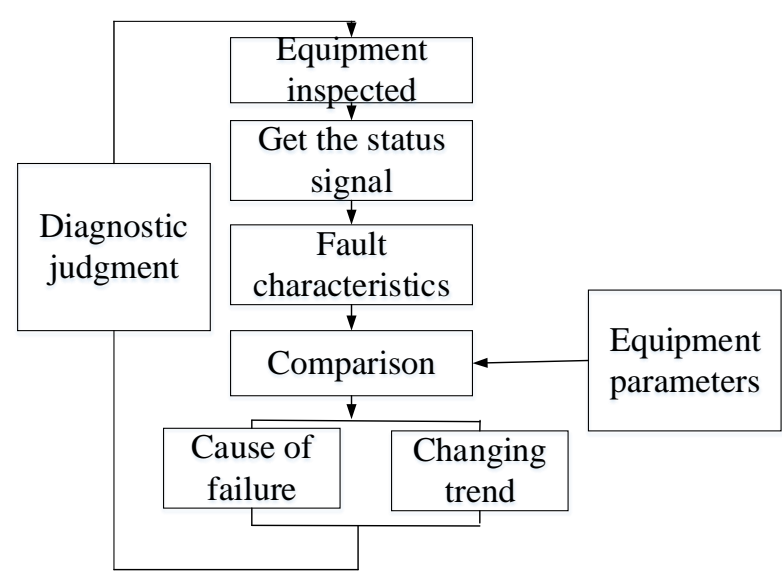

a)
Not only the control layer is added, but also the functions of function layer and data layer are expanded.

This structure not only has the excellent characteristics of $\mathrm{B} / \mathrm{S}$ mode, but also adds the function of the control service layer, making full use of the existing network resources and industrial field resources. And it organically combines the two to achieve the real-time monitoring based on $\mathrm{B} / \mathrm{S}$ remote mode.

\subsection{Basic Process of Rotating Machinery Failure Diagnosis}

For rotating machinery, various ways can be used to find their abnormal phenomena in operation, such as testing vibration, noise, temperature, and wear debris. Appropriate maintenance measures can be taken to prevent the occurrence of destructive accidents. The use of technology to achieve equipment remote monitoring increasingly becomes an indispensable step in the equipment monitoring system. The remote monitoring and diagnosis process of rotating machinery is shown in Figure 3.

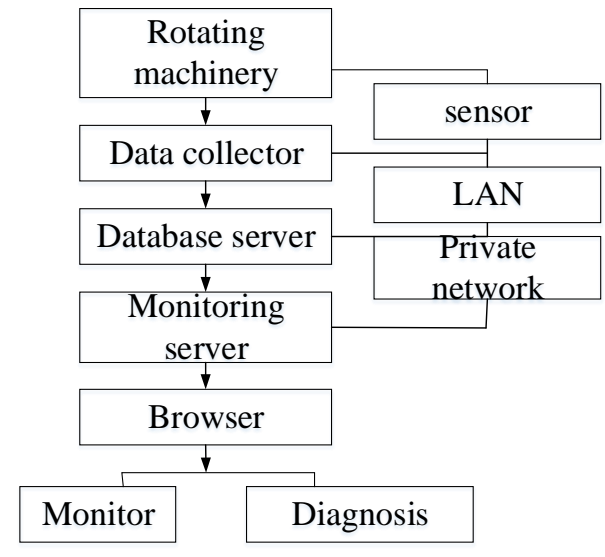

b)

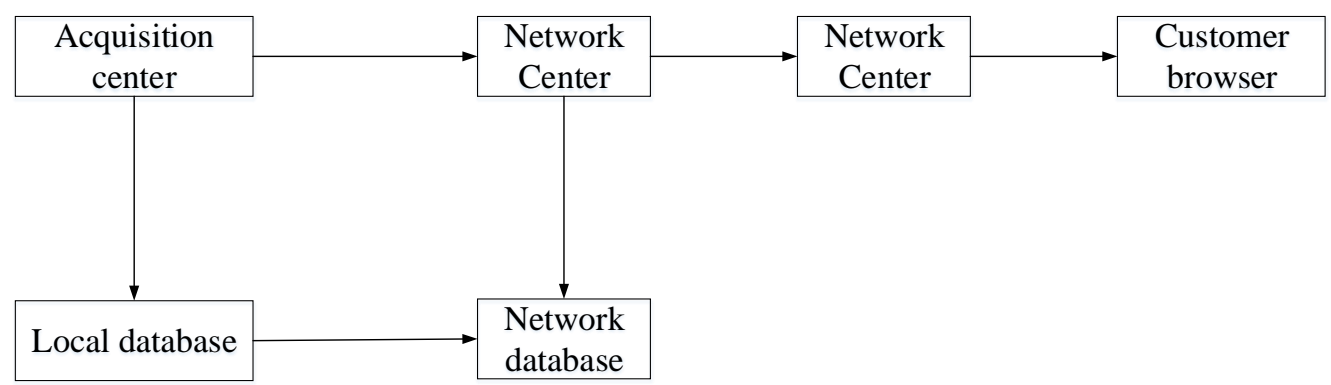

c)

Figure 2 Frame diagram. A) Failure diagnosis. B) Remote monitoring. C) Diagnosis system

Figure a) shows the failure diagnosis of rotating machinery: the first is to find out the location of the equipment failure; the other is to analyze the cause of the failure.
The failure diagnosis of rotating machinery mainly includes the following steps: acquisition of state signal, extraction of failure feature, recognition of mode state, failure diagnosis and diagnosis 
decision-making, of which the most important step is feature extraction of failure signal.

The specific steps are as follows: (1) In the actual production and operation of rotating machinery, there are often changes in force, temperature, vibration, noise and other qualities. According to the different physical qualities and equipment of diagnosis, it is necessary to select the corresponding sensor to obtain the relevant signal. (2) In the actual work, it is unreasonable to judge the running state of the failure equipment directly through the obtained characteristic signal. It needs to process the obtained state signal, like classification and processing, to extract the failure characteristics of the failure equipment. Different signal processing methods are the key to extracting failure features. (3) In the process of diagnosis, in order to confirm whether there is failure in the running equipment and identify the type and nature of the failure, it is necessary to compare the failure features extracted in step 2 with the limited data range of the specified equipment [11].

The step is usually performed by relevant experts before intelligent technology is introduced into the field of failure diagnosis. The rapid development of artificial intelligence promotes the application of knowledge-based diagnosing and reasoning in the field. (4) Diagnosis decision is to make correct and reasonable decision according to the location and nature of mechanical failure and the trend of malignant failure after the state identification step is completed to reduce and avoid the equipment deterioration and the economic loss of the enterprise. Figure b) indicates that the rotating machinery collects all kinds of data from the industrial control system and puts them into the database, and then transmits the corresponding data to the browser of the client and displays it on the screen, which is an important step of the remote monitoring equipment.

The remote monitoring system is composed of data acquisition and processing, database server, monitoring server and remote client. It is used as a communication network to realize the interaction between remote client and monitoring server. Figure c) reflects that the first thing to start the system is to initialize the system, find the data acquisition equipment and confirm whether it is connected normally. If the data acquisition module is in a good condition, the sampling parameters of the signal will be set as required. After the preliminary setup required by the system is finished, failure vibration signal is started to collect and sent to the industrial computer regularly. Subsequently, the extracted failure information is compared with the failure information in the database to determine the failure type.

\subsection{Overall Design Scheme of Diagnosis System}

The design scheme is to use the database to collect the vibration signals of various frequencies in the rotating machinery. The signal data are collected by the data acquisition card, and then the failure vibration signals are processed by using the software designed by the system to achieve the purpose of failure diagnosis. Rotating machinery failure diagnosis system is a virtual instrument system, which combines software and hardware, so the diagnosis system designed includes hardware and software. The foundation is the hardware, and the core of the system is the software, which plays a decisive role in the operation. The overall structure of rotating machinery failure diagnosis system is shown in Figure 4.

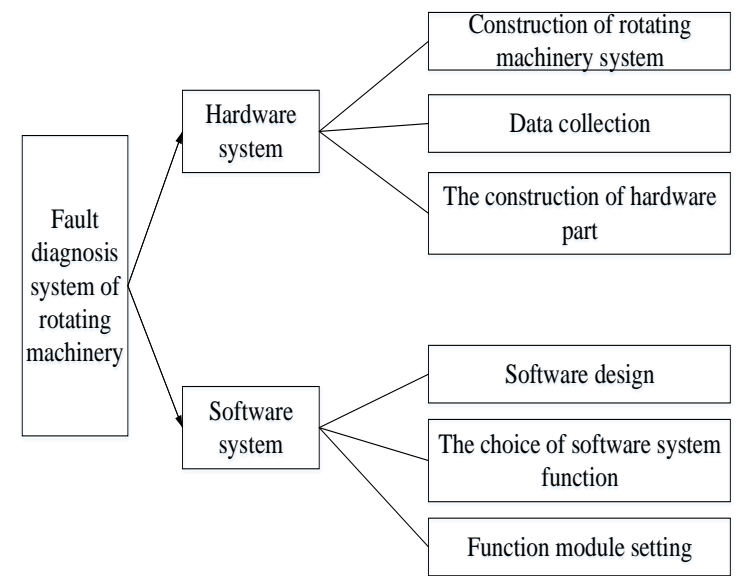

Figure 3: Design of rotating machinery failure diagnosis

The composition of hardware system of rotating machinery failure diagnosis system is shown in Figure 5. The hardware system includes mechanical system, sensor, anti-interference circuit/signal conditioning circuit, data acquisition equipment, required interface and supporting data connection line.

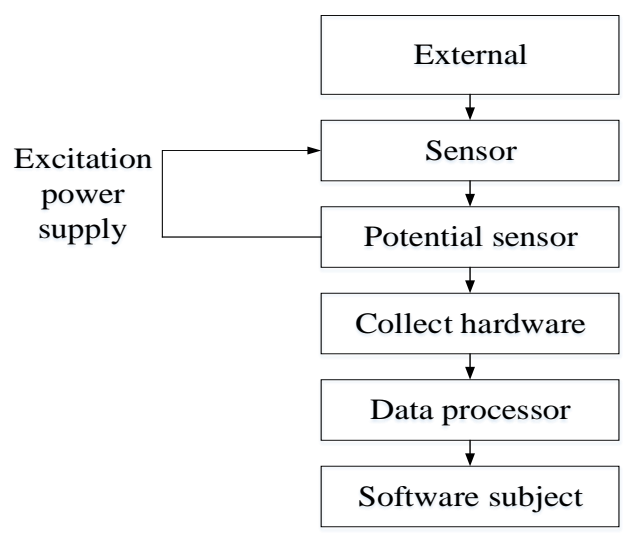

Figure 4: Composition of hardware system 
The hardware system includes mechanical system, sensor, anti-interference circuit/signal conditioning circuit, data acquisition equipment, required interface and supporting data connection line.

\subsection{Simulation Experiment}

The failure simulation experiment of rotating machinery is based on the comprehensive test bed for power transmission failure diagnosis especially designed by SpectraQuest, which can simulate industrial power transmission. The experimental platform consists of a motor control device, a twostage planetary gearbox, a driving motor, a two-stage parallel gearbox supported by rolling bearing and a magnetic brake. The test hardware of the experiment includes vibration acceleration sensor, tachometer, collector and host. PCB352C03 piezoelectric acceleration sensor is selected because of its good stability, reliable use, high precision, strong antiinterference ability and wide application range, with $1000 \mathrm{mv} / \mathrm{g}$ sensitivity and $5 \mathrm{~g}$ range [12].

The tachometer is the photoelectric speed sensor of the experimental platform.

The whole test device has 8 measuring points to obtain vibration signals from each major position of the equipment to measure adequate data for later research [13]. Under the consistent test circumstance, each situation is divided into three different speeds, and four groups of data are tested respectively to obtain as much experimental data as possible.

The specific distribution of measuring points is shown in the figure. And the general principles of measuring point selection are: (1) select the key positions of equipment, such as vibration sensitive points, vulnerable points; (2) the measuring points should be close to the measured object as far as possible to avoid the interference in the transmission process; (3) the measuring points should be located in the place with high stiffness to ensure the transmission of signal and avoid the signal weakening. The positions of each measuring point are shown in Table 1.

Table1 Distribution of measuring points

\begin{tabular}{|c|c|c|c|}
\hline $\begin{array}{l}\text { Measuring } \\
\text { point No. }\end{array}$ & measuring object & sensor position & sensor type \\
\hline A & $\begin{array}{c}\text { planetary gearbox input X } \\
\text { direction }\end{array}$ & \multirow[t]{2}{*}{ Bearing end cover radial } & \multirow{8}{*}{ РCB352C03 } \\
\hline B & $\begin{array}{c}\text { planetary gearbox input Y } \\
\text { direction }\end{array}$ & & \\
\hline $\mathrm{C}$ & $\begin{array}{c}\text { planetary gearbox output } \mathrm{X} \\
\text { direction }\end{array}$ & \multirow[t]{2}{*}{ Bearing end cover radial } & \\
\hline $\mathrm{D}$ & $\begin{array}{c}\text { planetary gearbox output } Y \\
\text { direction }\end{array}$ & & \\
\hline E & $\begin{array}{l}\text { parallel gearbox input X } \\
\text { direction }\end{array}$ & \multirow[t]{2}{*}{ Bearing end cover radial } & \\
\hline $\mathrm{F}$ & $\begin{array}{c}\text { parallel gearbox input Y } \\
\text { direction }\end{array}$ & & \\
\hline G & parallel gearbox output X & \multirow[b]{2}{*}{ Bearing end cover radial } & \\
\hline $\mathrm{H}$ & $\begin{array}{l}\text { parallel gearbox output Y } \\
\text { direction }\end{array}$ & & \\
\hline
\end{tabular}

The problems often appear on the planetary gearbox and the parallel gearbox, so the arrangement of measuring points should be around them. No.1, No.2 and No.4 measuring points are mainly used to test the X-Y vibration acceleration signal of the input end of the planetary gearbox, and No.3 and No.4 points are used to test the X-Y vibration acceleration signal of the output end of the planetary gearbox. No. 5 and No. 6 are arranged near the intermediate shaft at the input end of the parallel gearbox, because the failure gear and the failure bearing are arranged on the intermediate shaft for testing the vibration acceleration signals in $\mathrm{X}-\mathrm{Y}$ direction respectively. No. 7 and No. 8 are located on the output end of the parallel gearbox to measure the vibration acceleration signal in X-Y direction output by the intermediate shaft. No.9 is the speed signal.

The photoelectric speed sensor is used to reflect a square wave signal every revolution to collect the speed signal.

\section{Implementation of Remote Monitoring and Failure Diagnosis 3.1 Dynamic Refresh of Monitoring Atlas}

Spectrum monitoring is an important technology in remote monitoring and failure diagnosis of rotating machinery. Figure 6 is the wave form of failure signals. 


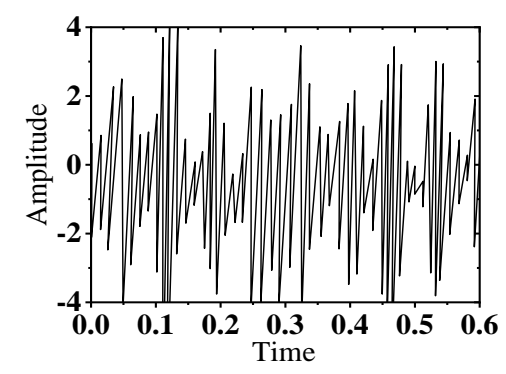

a)

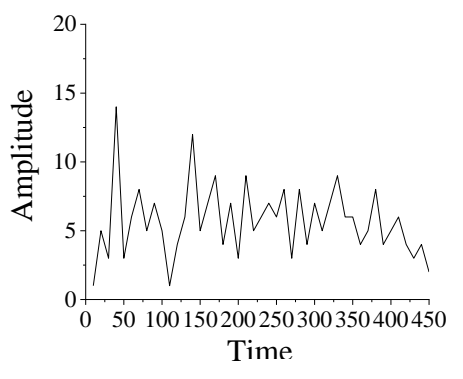

b)

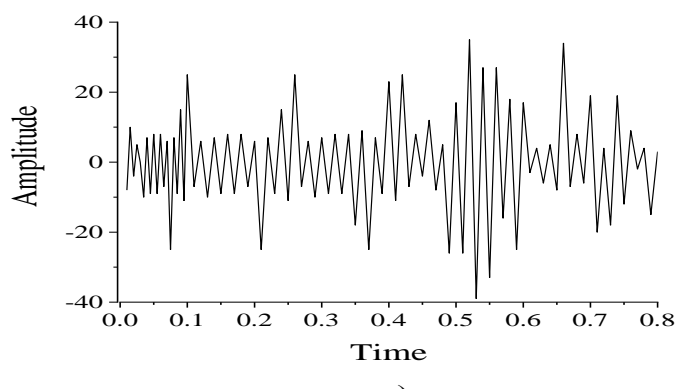

c)

Figure 5: Signal waveform. A) Inner ring failure. B) Vibration frequency. C) Vibration time

Figure a) demonstrates that the amplitude of the failure signal is high and low, which corresponds to the first and second times of the inner ring failure frequency, and the two failure frequencies are used to calculate theoretical values. Figure b) and c) are the results of outer ring failure signal through technical decomposition. Six different IMF (intrinsic mode function) components can show the characteristics of outer ring failure signal at different resolutions.

The bar monitoring graph displays the operation status of the whole equipment by monitoring the characteristic values of each channel, which can intuitively monitor the alarm position of each measuring point [14]. Multi value bar graph is to show the peak-to-peak value of the monitoring channels and each octave amplitude in the form of bar graph simultaneously. The real-time multi value bar monitoring graph is shown in Figure 7.

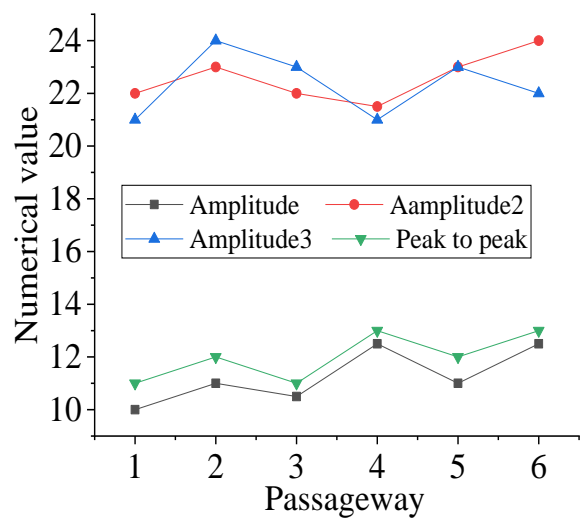

Figure 6: Multi value
There are four vertical bars with different colors in each vibration channel, which represent the amplitudes and peaks of the first, second and third harmonic generation respectively. The channel name is displayed at the bottom of each multi valued bar graph. If the number of channels increases, more passageways will be displayed to turn the pages through ChartPageNavigator. There is a TeeListBox component on the left side of the multi value bar graph to display one or more monitoring type values in multi value bar graph monitoring system.

At present, there are many ways to refresh the client page in the Web-based remote monitoring and diagnosis system, which can be divided into two kinds. (1) "Push" means that the server sends messages to each client, indicating that there is updated data. One way is to use the Socket port of the server to monitor and notify the client when there is data update [15]. But this way is only easy to realize in Local Area Network, but it is difficult to realize $B / S$ structure with agent on Internet.

The other way is to use the characteristics of database itself, such as trigger, to inform the client to update information. In two-tier system, the communication mode focusing on database server does not destroy the real-time performance greatly with the single server. However, in the three-tier or multi-tier, the effect of real-time refresh will be very poor when it is related to the database. Because in multi-layer, there are multiple database servers and application servers, and all kinds of servers will contact each other [16]. If the trigger is used to update the data, the change of one database server may be unknown to the change of the other database server, so the refresh and revisit mechanism of the 
database server binding must lead to a lot of extra work.

Since the remote equipment monitoring and diagnosis system is distributed, there are multiple devices from multiple monitoring branches in the monitoring field, accordingly there are multiple web servers and database servers [17].

In this case, this "push" method is not applicable to this system. (2) The data displayed in "pull" mode can be requested from the database in a certain way to realize real-time refresh. A common way to reach the request is to introduce a time control in the client, set the refresh time, and get real-time refresh.

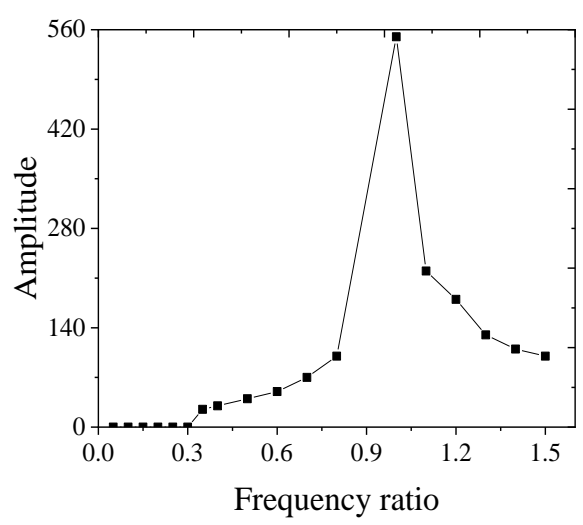

a)
And the whole page is also updated [18]. For the monitoring and diagnosis page, it needs to update the data in the page control.

However, the whole page needs to be downloaded from the server again, which causes a waste of resources and increases the burden of the server.

\subsection{Rotor Vibration Phase Analysis}

The amplitude frequency and phase frequency characteristics of the rotor are obtained by numerical integration method, as shown in Figure 8.

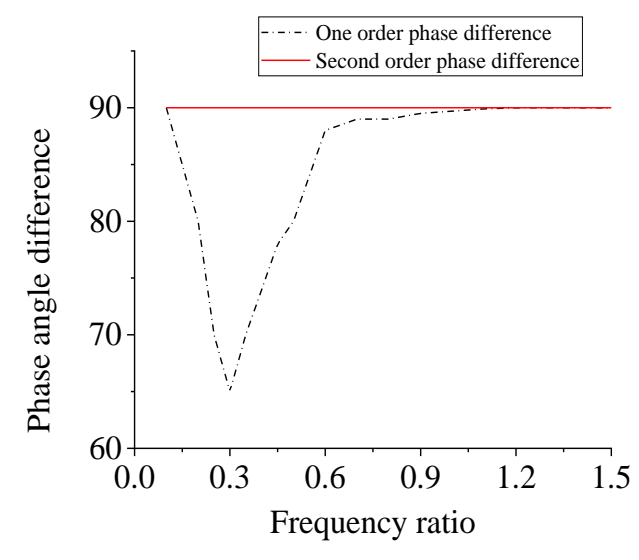

b)

Figure 7: Rotor calculation characteristics. A) Amplitude frequency. B) Phase frequency.

The abscissa is the frequency ratio. When the frequency ratio is $1 / 2$ and $1 / 3$, there is obvious vibration peak. When the frequency ratio is less than 1 and the phase difference is less than 90 degrees, there is a phase difference in the first order and 90 degrees in the second order.

Generally, only the magnitude and frequency of vibration are noticed in vibration monitoring and analysis, which is the case with conventional power spectrum analysis. These parameters are not applicable to some failures and are difficult to identify. Another applicable parameter is needed in failure analysis. Here it is easy to turn to the phase angle difference as a vibration parameter [19].

In essence, the phase angle difference is difficult to calculate, for the direction of the interference force and the damping is uncertain. The phase angle difference proposed here refers to the difference between the measured vibration displacement, vibration velocity or vibration acceleration signals of two points. The phase angle difference of the failure rotor is compared with that of the normal rotor to judge whether the rotor system is in failure and what failure occurs [20].
In order to obtain clear and accurate phase information, two vertical and horizontal measuring points on the vibration sensitive section of the shaft should be selected as a reference value, offering a comparative standard for the analysis of the failure rotor. Vibration signal usually contains many frequency components, which are defined as characteristic frequencies and their phases are characteristic phases.

\section{Discussion}

$\mathrm{B} / \mathrm{S}$ structure system is a change or improvement of C/S structure with the development of technology. It greatly simplifies the client computer load, reduces the cost and workload of system maintenance and upgrade, and decreases the overall cost of users. It can effectively protect the data platform, manage the access rights, and keep the database on the server safe.

Especially after the emergence of such crossplatform language, structure management software is more convenient, fast and efficient. Fan and Qian (2018) propose a data collection system based on 
$\mathrm{B} / \mathrm{S}$ structure. In $\mathrm{B} / \mathrm{S}$ mode, the client only needs a browser, and the browser interacts with the database through the web server [21]. The online shopping system is based on this mode because of such a huge advantage.

The system integrates the online shopping system through MVC (model, view, and controller), completes the management of control layer and offers the access to data. Thus, the system can basically meet the requirements of users, and it is also a good electronic application. Users can know the relevant information of products at any time and get a large amount of information. In addition, it completely solves the disadvantages of the traditional ones, such as the space and time limitation. Aiming at the problems of complex structure, diverse components and remote network diagnosis of rotating machinery, rotating machinery failure diagnosis system based on $\mathrm{B} / \mathrm{S}$ structure is recommended.

The design of remote real-time monitoring and failure intelligent diagnosis system of rotating machinery is discussed based on B/S mode to respond to the complex structure of rotating machinery, large vibration noise, remote network monitoring and diagnosis, and the problem that the historical failure data cannot be well used based on single- segment and signal-analysis method. The collection and reuse of historical failure data are realized by establishing failure vibration database.

\section{Conclusions}

The principle of remote real-time monitoring system of rotating machinery based on B / S mode is analyzed, its structure model is established, and its function structure and specific implementation mode are clarified. And the network structure of remote monitoring system of asphalt concrete mixing equipment based on B/S mode is designed, combining network technology, database and technology and dynamic web technology together.

After compared with various dynamic web page implementation technologies, the design is completely based on $\mathrm{B} / \mathrm{S}$ mode, and solves the platform inconsistency caused by the diversification of multi-user and the complexity of developing different client programs, so that multiple users can monitor the production remotely without space constraints, and it has strong practicability. On the basis of $\mathrm{B} / \mathrm{S}$ structure, the research of database module and failure diagnosis function module are integrated, creating a failure diagnosis method, which basically realizes the remote monitoring and intelligent diagnosis of rotating machinery based on B/S mode.

Although some research on the remote condition monitoring and diagnosis system is done, the system still needs to be further improved in some aspects. For example, the transmission of real-time data is affected due to unknown factors when the data pass through the Internet due to unknown factors, and the remote transmission data needs to be compressed more effectively.

\section{References}

[1] He K., Yang Z., Bai Y, et al. (2018) Intelligent Fault Diagnosis of Delta 3D Printers Using Attitude Sensors Based on Support Vector Machines. Sensors, 18(4), 1298.

[2] He D. (2019) Design and Implementation of Resource Information Management Based on B/S Structure. Journal of Physics Conference Series, 1345, 52028.

[3] Chen X., Wang S., Qiao B, et al. (2018) Basic research on machinery fault diagnostics: Past, present, and future trends. Frontiers of Mechanical Engineering, 13(2), 264-291.

[4] Chen M., Zhou Y., Huang X, et al. (2021) The Integration of New-Type Urbanization and Rural Revitalization Strategies in China: Origin, Reality and Future Trends. Land, 10(2), 1-17.

[5] Thobiani F A., Tran V T., Tinga T. (2017) An Approach to Fault Diagnosis of Rotating Machinery Using the Second-Order Statistical Features of Thermal Images and Simplified Fuzzy ARTMAP. Engineering, 9(6), 524-539.

[6] Luca M., Lorenzo G., Sarah H, et al. (2017) Remote detection of rotating machinery with a portable atomic magnetometer. Applied Optics, 56(3), 743.

[7] Te H., Dong X, et al. (2017) Comparison of random forest, artificial neural networks and support vector machine for intelligent diagnosis of rotating machinery. Transactions of the Institute of Measurement and Control, 40(8), 2681-2693.

[8] Han T., Liu C., Yang W, et al. (2019) A novel adversarial learning framework in deep convolutional neural network for intelligent diagnosis of mechanical faults. 165(1), 474-487.

[9] Tang S., Yuan S., Zhu Y. (2020) Cyclostationary Analysis towards Fault Diagnosis of Rotating Machinery. Processes, 8(10), 1217.

[10] Sathik M., Prasanth S., Sasongko F, et al. (2019) Online condition monitoring of IGBT modules using current-change rate identification. Microelectronics \& Reliability, 92, 55-62. 
[11] Alyushin M., Kolobash L. (2019) Monitoring of the current status of students as a means of increasing the effectiveness of educational process. The Education and science journal, 21(2), 176-197.

[12] Skopets 0., Korotkyi V. (2019) Stand for the System of Remote Analysis of Heart Rate Variability. Microsystems Electronics and Acoustics, 24(4), 48-55.

[13] Ma Z., Wang S., Shen J, et al. (2019) Design of multi-energy joint optimization dispatching system for regional power grids based on B/S architecture. Energy Procedia, 158, 6236-6241.

[14] Chai J. (2017) Development of Computer English Education Platform Based on B / S Model. International Journal of Emerging Technologies in Learning, 12(8), 86.

[15] Velichenko A., Shmychkova O., Samiolo L, et al. (2021) Reduction of nitroaromatics on cadmium sulfide: further probing the electrochemical model of semiconductor photocatalysis. Journal of Solid State Electrochemistry, 25(1), 85-92.

[16] Li Y., Gu J X., Zhen D, et al. (2019) An Evaluation of Gearbox Condition Monitoring Using Infrared Thermal Images Applied with Convolutional Neural Networks. Sensors, 19(9), 2205.
[17] Te H., Jiang D., Zhang X, et al. (2017) Intelligent Diagnosis Method for Rotating Machinery Using Dictionary Learning and Singular Value Decomposition. Sensors, 17(4), 689.

[18] Latif J., Khan Z A., Nazir M H, et al. (2018) Condition monitoring and predictive modelling of coating delamination applied to remote stationary and mobile assets. Structural Health Monitoring, 18(4), 1056-1073.

[19] Peng Y., Qiao W., Qu L, et al. (2018) Sensor Fault Detection and Isolation for a Wireless Sensor Network-Based Remote Wind Turbine Condition Monitoring System. IEEE Transactions on Industry Applications, 54(2), 1072-1079.

[20] Kaliaskarov N., Ivel V., Gerasimova Y, et al. (2020) Development of a distributed wireless Wi-Fi system for monitoring the technical condition of remote objects. Eastern-European Journal of Enterprise Technologies, 5(9), 36-48.

[21] Fan W., Qian Z. (2018) Design and Implementation of Online Shopping System Based on B/S Model. MATEC Web of Conferences, 246, 26-29. 